PROCEEDINGS OF THE

AMERICAN MATHEMATICAL SOCIETY

Volume 127, Number 10, Pages 2987-2994

S 0002-9939(99)04886-8

Article electronically published on April 28, 1999

\title{
ON POINTWISE CONVERGENCE OF FOURIER SERIES OF RADIAL FUNCTIONS IN SEVERAL VARIABLES
}

\author{
SHIGEHIKO KURATSUBO
}

(Communicated by Frederick W. Gehring)

\begin{abstract}
We prove the pointwise convergence of the Fourier series for radial functions in several variables, which in the case $n=1$ is the Dirichlet-Jordan theorem itself. In our proof the method for the case of the indicator function of the ball is very useful.
\end{abstract}

\section{INTRODUCTION}

$Z^{n}$ denotes the $n$ dimensional integer lattice, whose points are written $m=$ $\left(m_{1}, \cdots, m_{n}\right)$, where $m_{k}$ are any integers. $R^{n}$ denotes the $n$ dimensional Euclidean spaces, whose points are written $x=\left(x_{1}, \cdots, x_{n}\right)$, and for $x, y \in R^{n}$, its inner product is $x y=\sum_{k=1}^{n} x_{k} y_{k} . Q^{n}$ denotes the set whose points are $x=\left(x_{1}, \cdots, x_{n}\right)$, where $x_{k}$ are any rational numbers.

We aim to consider the pointwise behavior of the Fourier series of some sort of bounded variation function in several variables.

The Fourier transform and its spherical partial sum of a function $f$ on $R^{n}$ are defined by

$$
\hat{f}(\xi):=\int_{R^{n}} f(x) e^{-2 \pi i \xi x} d x \quad\left(\xi \in R^{n}\right)
$$

and

$$
f_{\lambda}(x):=\int_{|\xi|<\lambda} \hat{f}(\xi) e^{2 \pi i \xi x} d \xi .
$$

Next, the Fourier series and its spherical sum of a function $F$ on $T^{n}$, where $T^{n}$ $=\left[-\frac{1}{2}, \frac{1}{2}\right]^{n}$, are defined by

$$
\hat{F}(m):=\int_{T^{n}} F(x) e^{-2 \pi i m x} d x \quad\left(m \in Z^{n}\right)
$$

and

$$
S_{\lambda}(F, x):=\sum_{|m|<\lambda} \hat{F}(m) e^{2 \pi i m x}
$$

Received by the editors September 30, 1997 and, in revised form, January 7, 1998.

1991 Mathematics Subject Classification. Primary 42B05.

Key words and phrases. Fourier series, spherical partial sum, bounded variation, indicator function, lattice point problem.

(C)1999 American Mathematical Society 
M. A. Pinsky and others [7], [8] considered the pointwise convergence of the Fourier inversion of radial functions in several variables and the convergence at $x=0$ of the Fourier series of the indicator function of the ball.

The indicator function of ball with radius $a>0$ and center 0 is defined by $\chi_{a}(x):=1$ for $|x| \leq a$ and $\chi_{a}(x):=0$ for $|x|>a$. Also we defined $\overline{\chi_{a}}(x):=1$ for $|x|<a, \overline{\chi_{a}}(x):=\frac{1}{2}$ for $|x|=a$, and $\overline{\chi_{a}}(x):=0$ for $|x|>a$. Then we have

$$
\hat{\chi_{a}}(\xi)= \begin{cases}a^{\frac{n}{2}} \frac{J_{\frac{n}{2}}(2 \pi|\xi| a)}{|\xi|^{\frac{n}{2}}} & \text { for } \xi \neq 0, \\ \frac{\pi^{\frac{n}{2}} a^{n}}{\Gamma\left(\frac{n}{2}+1\right)} & \text { for } \xi=0\end{cases}
$$

and

$$
\chi_{a, \lambda}(x)= \begin{cases}\left(\frac{a}{|x|}\right)^{\frac{n}{2}-1} \int_{0}^{2 \pi a \lambda} J_{\frac{n}{2}}(s) J_{\frac{n}{2}-1}\left(\frac{|x|}{a} s\right) d s, & \text { for } x \neq 0, \\ \frac{1}{2^{\frac{n}{2}-1} \Gamma\left(\frac{n}{2}\right)} \int_{0}^{2 \pi a \lambda} s^{\frac{n}{2}-1} J_{\frac{n}{2}}(s) d s, & \text { for } x=0,\end{cases}
$$

where $J_{\alpha}$ is the Bessel function of the first kind of order $\alpha$.

Lemma 1. Suppose $a>0$ and $\varphi$ is a function of bounded variation, and

$$
f_{\varphi}(x):= \begin{cases}\varphi(t) & \text { for }|x|=t<a \\ 0 & \text { for }|x|>a\end{cases}
$$

Then we have

$$
\hat{f}_{\varphi}(\xi)=\varphi(a) \hat{\chi_{a}}(\xi)-\int_{0}^{a} \hat{\chi_{t}}(\xi) d \varphi(t),
$$

where $d \varphi$ is the Lebesgue-Stieltjes measure generated by $\varphi$.

Proof.

$$
\begin{gathered}
\hat{f}_{\varphi}(\xi)=\int_{0}^{a} \varphi(t) t^{n-1}\left(\int_{\Sigma_{n-1}} e^{2 \pi i t|\xi| x^{\prime} \xi^{\prime}} d \sigma_{x^{\prime}}\right) d t \\
=2 \pi \int_{0}^{a} \varphi(t) t^{n-1} \frac{J_{\frac{n}{2}-1}(2 \pi t|\xi|)}{(t|\xi|)^{\frac{n}{2}-1}} d t \\
=\frac{2 \pi}{|\xi|^{\frac{n}{2}-1}} \int_{0}^{a} \varphi(t) t^{\frac{n}{2}} J_{\frac{n}{2}-1}(2 \pi t|\xi|) d t=\frac{1}{|\xi|^{\frac{n}{2}}} \int_{0}^{a} \varphi(t) \frac{d}{d t}\left(t^{\frac{n}{2}} J_{\frac{n}{2}}(2 \pi t|\xi|)\right) d t \\
=\varphi(a) a^{\frac{n}{2}} \frac{J_{\frac{n}{2}}(2 \pi a|\xi|)}{|\xi|^{\frac{n}{2}}}-\frac{1}{|\xi|^{\frac{n}{2}}} \int_{0}^{a} t^{\frac{n}{2}} J_{\frac{n}{2}}(2 \pi t|\xi|) d \varphi(t) \\
=\varphi(a) \hat{\chi_{a}}(\xi)-\int_{0}^{a} \hat{\chi_{t}}(\xi) d \varphi(t) .
\end{gathered}
$$

This completes the proof of our lemma.

Next, we introduce two periodic functions $F_{t}$ and $F_{\varphi}$ with respect to each coordinate with period 1 :

$$
F_{t}(x):=\sum_{m \in Z^{n}} \chi_{t}(x+m)
$$


and

$$
F_{\varphi}(x):=\sum_{m \in Z^{n}} f_{\varphi}(x+m) .
$$

In particular, in the case of $0<t<\frac{1}{2}, F_{t}$ is equal on $T^{n}$ to the indicator function of the ball with radius $t$ and center 0 .

Then we have the following lemma.

\section{Lemma 2.}

$$
S_{\lambda}\left(F_{\varphi}: x\right)=\varphi(a) S_{\lambda}\left(F_{a}: x\right)-\int_{0}^{a} S_{\lambda}\left(F_{t}: x\right) d \varphi(t) .
$$

Proof. Since $\hat{F}_{t}(m)=\hat{\chi}_{t}(m)$ and $\hat{F}_{\varphi}(m)=\hat{f}_{\varphi}(m)$ by the Poisson Summation Formula, we have by Lemma 1

$$
\hat{F}_{\varphi}(m)=\varphi(a) \hat{\chi_{a}}(m)-\int_{0}^{a} \hat{\chi_{t}}(m) d \varphi(t) .
$$

Therefore we have

$$
\begin{gathered}
S_{\lambda}\left(F_{\varphi}: x\right)=\varphi(a) \sum_{|m|<\lambda} \hat{\chi_{a}}(m) e^{2 \pi i m x}-\int_{0}^{a}\left(\sum_{|m|<\lambda} \hat{\chi}_{t}(m) e^{2 \pi i m x}\right) d \varphi(t) \\
=\varphi(a) S_{\lambda}\left(F_{a}: x\right)-\int_{0}^{a} S_{\lambda}\left(F_{t}: x\right) d \varphi(t) .
\end{gathered}
$$

This completes the proof of Lemma 2 .

The aim of this paper is to consider the behavior of $S_{\lambda}\left(F_{\varphi}: x\right)$. Some related papers are Hardy-Landau [3], Pinsky, Stanton and Trapa [8], Pinsky [7], Kuratsubo [4], and L. Brandolini and L. Colzani [1].

Theorem (Hardy-Landau [3]). If $n=2$ and $a>0$, then

$$
\lim _{\lambda \rightarrow \infty}\left\{\pi a+a \sum_{0<|m|<\lambda} \frac{J_{1}(2 \pi a|m|)}{|m|}\right\}=\sum_{|m|<a} 1+\frac{1}{2} \sum_{|m|=a} 1 .
$$

Theorem (Pinsky, Stanton and Trapa [8]). If $0<a<\frac{1}{2}$ and $n \geq 3$, then the Fourier series of the indicator function of the ball $|x| \leq a$ diverges at center $x=0$.

The results of Hardy-Landau [3] show that in the case of $n=2$ the Fourier series of the indicator function of the ball $|x| \leq a$ converges to $\overline{\chi_{a}}(0)$ at center $x=0$.

Pinsky and others [8] noted the equality:

$$
\begin{gathered}
\sum_{|m| \leq \lambda} \hat{\chi_{a}}(m)=\omega_{n-1} \int_{0}^{\lambda} \Lambda(s) s^{n-1} d s \\
+\Lambda(\lambda)\left(\sum_{|m| \leq \lambda} 1-v_{n} \lambda^{n}\right)-\int_{0}^{\lambda} \Lambda^{\prime}(s)\left(\sum_{|m| \leq s} 1-v_{n} s^{n}\right) d s,
\end{gathered}
$$

where $\omega_{n-1}, v_{n}$ are the area of the $n$ dimensional sphere and the volume of the $n$ dimensional ball and

$$
\Lambda(s)=a^{\frac{n}{2}} \frac{J_{\frac{n}{2}}(2 \pi a s)}{s^{\frac{n}{2}}}
$$


Then, the first term is the leading one and the estimation of $\sum_{|m| \leq \lambda} 1-v_{n} \lambda^{n}$ is the lattice-points problem itself. The method of Kuratsubo [4] originates from the idea of Hardy-Landau.

Theorem (Kuratsubo [4]). For $x \neq 0$ we have the following:

$$
\begin{aligned}
& \text { If } 2 \leq n \leq 4, \lim _{\lambda \rightarrow \infty} S_{\lambda}\left(F_{a}: x\right)=\overline{F_{a}}(x) . \\
& \text { If } n \geq 5 \text { and } x \notin Q^{n}, \limsup _{\lambda \rightarrow \infty}\left(\lambda^{\frac{5-n}{2}}\left|S_{\lambda}\left(F_{a}: x\right)-\overline{F_{a}}(x)\right|\right)=0 . \\
& \text { If } n \geq 5 \text { and } x \in Q^{n}, \limsup _{\lambda \rightarrow \infty}\left(\lambda^{\frac{7-n}{2}}\left|S_{\lambda}\left(F_{a}: x\right)-\overline{F_{a}}(x)\right|\right)<\infty . \\
& \text { If } n \geq 6, \lim _{\lambda \rightarrow \infty} S_{\lambda}\left(F_{a}: x\right)=\overline{F_{a}}(x) \text { for almost all } x .
\end{aligned}
$$

The main results of our paper are the following Theorems 1,2 and 3. These theorems are extensions of Kuratsubo [4] from the case of the indicator function of a ball to the more general case of radial functions of bounded variation.

Theorem 1. If $1 \leq n \leq 4$, then for $x \neq 0$, we have

$$
\begin{gathered}
\lim _{\lambda \rightarrow \infty} S_{\lambda}\left(F_{\varphi}: x\right)=\varphi(a) \overline{F_{a}}(x)-\int_{0}^{a} \overline{F_{t}}(x) d \varphi(t) \\
=\sum_{|x+m|<a} \frac{\varphi(|x+m|+)+\varphi(|x+m|-)}{2}+\frac{\varphi(a)+\varphi(a-)}{2} \sum_{|x+m|=a} 1 .
\end{gathered}
$$

Theorem 2. If $n=5$, then for any $x \notin Q^{n}$, we have $(*)$.

Theorem 3. If $n \geq 6$, for almost everywhere $x$, we have (*).

\section{Some Results on LATtice Point PRoblems}

A key to our methods is in lattice point problems. On these problems Fricker [2] is very thorough. Our interest is to estimate the following:

$$
P_{\alpha}(t: x):=K_{\alpha}(t: x)-\frac{\pi^{\frac{n}{2}} t^{\frac{n}{2}+\alpha}}{\Gamma\left(\frac{n}{2}+\alpha+1\right)} \delta(x),
$$

where $\alpha \geq 0$,

$$
K_{\alpha}(t: x):=\frac{1}{\Gamma(\alpha+1)} \sum_{|m|^{2}<t}\left(t-|m|^{2}\right)^{\alpha} e^{2 \pi i m x},
$$

and $\delta(x):=1$ if $x \in Z^{n}$ and $\delta(x):=0$ otherwise. These problems have been studied by Landau, Jarník, Szegö, Novák and others. The important results are the following:

Theorem (Landau [5]). If $n \geq 2$ and $x \in R^{n}$, then

$$
P_{\alpha}(t: x)= \begin{cases}O\left(t^{\frac{n-1}{4}+\frac{\alpha}{2}}\right) & \text { if } \alpha>\frac{n-1}{2}, \\ O\left(t^{\frac{n-1}{4}+\frac{\alpha}{2}} \log t\right) & \text { if } \alpha=\frac{n-1}{2}, \\ O\left(t^{\frac{n}{2}+\alpha-\frac{n}{n+1-2 \alpha}}\right) & \text { if } 0 \leq \alpha<\frac{n-1}{2} .\end{cases}
$$


Theorem (Novák [6]). If $0 \leq \alpha<\frac{n}{2}-2$, then

$$
P_{\alpha}(t: x)= \begin{cases}O\left(t^{\frac{n}{2}-1}\right), \Omega\left(t^{\frac{n}{2}-1}\right) & \text { for } x \in Q^{n}, \\ o\left(t^{\frac{n}{2}-1}\right) & \text { for } x \notin Q^{n}, \\ O\left(t^{\frac{n}{4}+\frac{\alpha}{2}} \log ^{\tau} t\right) & \text { for almost all } x,\end{cases}
$$

where $\tau=3 n-1$ if $\alpha>0$ and $\tau=3 n$ for $\alpha=0$.

The proofs of our main theorems need the following lemmas. Let us define by $k_{0}$ the smallest of integers $k$ such that it satisfies the inequality: $\frac{n-1}{2}<k$.

Lemma 3. If $n \geq 2$,

$$
P_{\alpha}(t: x)=O\left(t^{\psi_{1}(\alpha)}\right) \quad \text { for } x \in R^{n} \text { and } 0 \leq \alpha \leq k_{0},
$$

where $\psi_{1}(\alpha)$ is the linear function tying two points: $\left(0, \frac{n}{2}-\frac{n}{n+1}\right),\left(k_{0}, \frac{n-1}{4}+\frac{k_{0}}{2}\right)$.

Lemma 4. If $n \geq 5$,

$$
P_{\alpha}(t: x)=O\left(t^{\psi_{2}(\alpha)}\right) \quad \text { for } x \in R^{n} \text { and } 0 \leq \alpha \leq k_{0},
$$

where $0<\varepsilon<\frac{1}{2}$ and $\psi_{2}(\alpha)$ is the linear curve tying three points: $\left(0, \frac{n}{2}-1\right)$, $\left(\frac{n}{2}-2-\varepsilon, \frac{n}{2}-1\right)$, and $\left(k_{0}, \frac{n-1}{4}+\frac{k_{0}}{2}\right)$.

Lemma 5. If $n \geq 5$,

$$
P_{\alpha}(t: x)=O\left(t^{\psi_{3}(\alpha)}\right) \quad \text { for almost all } x \text { and } 0 \leq \alpha \leq k_{0},
$$

where $\varepsilon>0$ and $\psi_{3}(\alpha)$ is the linear function tying two points: $\left(0, \frac{n}{4}+\varepsilon\right)$ and $\left(k_{0}, \frac{n-1}{4}+\frac{k_{0}}{2}\right)$.

The proofs of these lemmas are derived from the above two theorems and Riesz's convexity theorem:

Riesz's convexity theorem. For any numerical sequence $\left\{c_{k}\right\}_{k=0}^{\infty}$, consider its Riesz means:

$$
\sigma_{t}^{\alpha}=\sum_{0 \leq k<t}\left(1-\frac{k}{t}\right)^{\alpha} c_{k}, \alpha \geq 0 .
$$

Suppose that $\sigma_{t}^{\alpha_{j}}=O\left(t^{a_{j}}\right)$ as $t \rightarrow \infty$, for $j=0,1$. Then,

$$
\text { if } 0<\theta<1, \alpha=\alpha_{0}(1-\theta)+\alpha_{1} \theta \text {, and } a=a_{0}(1-\theta)+a_{1} \theta \text {, }
$$

we can conclude that $\sigma_{t}^{\alpha}=O\left(t^{a}\right)$ as $t \rightarrow \infty$.

(See Stein-Weiss [9], p. 285.)

\section{Proofs of MAIN THEOREMS}

The starting point of our proofs is the following theorem.

Theorem (Kuratsubo [4]). Suppose $k_{0}$ is the smallest of integers $k$ which satisfies $k>\frac{n-1}{2}$; we have

$$
\begin{aligned}
& S_{\lambda}\left(F_{t}: x\right)=\overline{F_{t}}(x)+\left(\chi_{t, \lambda}(0)-1\right) \delta(x)+t^{\frac{n}{2}} \sum_{l=0}^{k_{0}}(\pi t)^{l} P_{l}\left(\lambda^{2}: x\right) \Lambda_{l}\left(t: \lambda^{2}\right) \\
& \quad+\sum_{m-x \neq 0}\left(\frac{t}{|m-x|}\right)^{\frac{n}{2}+k_{0}} \int_{2 \pi t \lambda}^{\infty} J_{\frac{n}{2}+k_{0}}\left(\frac{|m-x|}{t} s\right) J_{\frac{n}{2}+k_{0}+1}(s) d s,
\end{aligned}
$$


where $\delta(x):=1$ if $x \in Z^{n}$ and $\delta(x):=0$ if $x \notin Z^{n}$, and

$$
\overline{F_{t}}(x):=\sum_{m} \overline{\chi_{t}}(m+x) \text { and } \Lambda_{\alpha}(t: \lambda):=\frac{J_{\frac{n}{2}+\alpha}(2 \pi t \sqrt{\lambda})}{\lambda^{\frac{n}{4}+\frac{\alpha}{2}}} .
$$

Furthermore, the last term is absolutely convergent with respect to $m$ and $o(1)$ as $\lambda \rightarrow \infty$.

(See Kuratsubo [4].)

Lemma 6. For any $x$, we have

$$
\begin{aligned}
& S_{\lambda}\left(F_{\varphi}: x\right)=\left[\left\{\varphi(a) \overline{F_{a}}(x)-\int_{0}^{a} \overline{F_{t}}(x) d \varphi(t)\right\}+\left\{\varphi(a)\left(\chi_{a, \lambda}(0)-1\right)\right.\right. \\
& \left.-\int_{0}^{a}\left(\chi_{t, \lambda}(0)-1\right) d \varphi(t)\right\} \delta(x)+\varphi(a) a^{\frac{n}{2}} \sum_{l=0}^{k_{0}}(\pi a)^{l} P_{l}\left(\lambda^{2}: x\right) \Lambda_{l}\left(a: \lambda^{2}\right) \\
& \left.-\sum_{l=0}^{k_{0}} P_{l}\left(\lambda^{2}: x\right) \int_{0}^{a} t^{\frac{n}{2}}(\pi t)^{l} \Lambda_{l}\left(t: \lambda^{2}\right) d \varphi(t)\right]+o(1) \quad \text { as } \lambda \rightarrow \infty .
\end{aligned}
$$

Proof. Since

$$
\begin{gathered}
\int_{0}^{a} S_{\lambda}\left(F_{t}: x\right) d \varphi(t)=\int_{0}^{a} \overline{F_{t}}(x) d \varphi(t) \\
+\left(\int_{0}^{a}\left(\chi_{t, \lambda}(0)-1\right) d \varphi(t)\right) \delta(x)+\sum_{l=0}^{k_{0}} P_{l}\left(\lambda^{2}: x\right) \int_{0}^{a} t^{\frac{n}{2}}(\pi t)^{l} \Lambda_{l}\left(t: \lambda^{2}\right) d \varphi(t) \\
+\sum_{m-x \neq 0} \int_{0}^{a}\left(\frac{t}{|m-x|}\right)^{\frac{n}{2}+k_{0}}\left\{\int_{2 \pi t \lambda}^{\infty} J_{\frac{n}{2}+k_{0}}\left(\frac{|m-x|}{t} s\right) J_{\frac{n}{2}+k_{0}+1}(s) d s\right\} d \varphi(t),
\end{gathered}
$$

we conclude the proof of Lemma 6 from the following lemma.

Throughout this section, the notation $A \ll B$ implies $|A| \leq C B$ for some absolute constant $C$.

Lemma 7. The infinite series

$$
\Sigma(\lambda):=\sum_{m-x \neq 0} \int_{0}^{a}\left(\frac{t}{|m-x|}\right)^{\frac{n}{2}+k_{0}}\left\{\int_{2 \pi t \lambda}^{\infty} J_{\frac{n}{2}+k_{0}}\left(\frac{|m-x|}{t} s\right) J_{\frac{n}{2}+k_{0}+1}(s) d s\right\} d \varphi(t)
$$

is absolutely convergent and

$$
\Sigma(\lambda)=o(1) \quad \text { as } \lambda \rightarrow \infty .
$$

Proof. Firstly in the case of $|m-x| \geq 2 a$,

$$
\begin{aligned}
& \left|\int_{0}^{a}\left(\frac{t}{|m-x|}\right)^{\frac{n}{2}+k_{0}}\left\{\int_{2 \pi t \lambda}^{\infty} J_{\frac{n}{2}+k_{0}}\left(\frac{|m-x|}{t} s\right) J_{\frac{n}{2}+k_{0}+1}(s) d s\right\} d \varphi(t)\right| \\
& \ll \int_{0}^{a}\left(\frac{t}{|m-x|}\right)^{\frac{n}{2}+k_{0}} \int_{2 \pi t \lambda}^{\infty}\left|J_{\frac{n}{2}+k_{0}}\left(\frac{|m-x|}{t} s\right) J_{\frac{n}{2}+k_{0}+1}(s) d s\right||d \varphi|(t)
\end{aligned}
$$




$$
\begin{aligned}
& \ll \int_{0}^{a}\left(\frac{t}{|m-x|}\right)^{\frac{n}{2}+k_{0}}\left(\frac{1}{\left(\frac{|m-x|}{t}\right)^{\frac{1}{2}} \mid} \int_{|| m-x|-t| 2 \pi \lambda}^{\infty} \frac{\sin s}{s} d s \mid\right. \\
& \left.+\frac{1}{\left(\frac{|m-x|}{t}\right)^{\frac{1}{2}}} \frac{1}{t \lambda}\left(1+\frac{1}{t \lambda}\right)\right)|d \varphi|(t) \\
& \ll \frac{1}{|m-x|^{\frac{n+1}{2}+k_{0}}} \frac{1}{\lambda} \int_{0}^{a}\left(t^{\frac{n+1}{2}+k_{0}}+t^{\frac{n-1}{2}+k_{0}}\left(1+\frac{1}{t \lambda}\right)\right)|d \varphi|(t) \\
& \ll \frac{1}{\lambda} \frac{1}{|m-x|^{\frac{n+1}{2}+k_{0}}} .
\end{aligned}
$$

From this estimation the series $\Sigma(\lambda)$ is absolutely convergent.

Secondly in the case of $|m-x|<2 a$,

$$
\begin{gathered}
\left|\left(\frac{t}{|m-x|}\right)^{\frac{n}{2}+k_{0}}\left\{\int_{2 \pi t \lambda}^{\infty} J_{\frac{n}{2}+k_{0}}\left(\frac{|m-x|}{t} s\right) J_{\frac{n}{2}+k_{0}+1}(s) d s\right\}\right| \\
\ll\left(\frac{t}{|m-x|}\right)^{\frac{n}{2}+k_{0}}\left(\frac{1}{\left(\frac{|m-x|}{t}\right)^{\frac{1}{2}}}+\left(\frac{|m-x|}{t}\right)^{\frac{n}{2}+k_{0}}\right) \\
\ll\left(\frac{t}{|m-x|}\right)^{\frac{n+1}{2}+k_{0}}+1 \ll\left(\frac{a}{|m-x|}\right)^{\frac{n+1}{2}+k_{0}}+1 \quad \text { for } 0 \leq t \leq a
\end{gathered}
$$

and

$$
\lim _{\lambda \rightarrow \infty}\left(\frac{t}{|m-x|}\right)^{\frac{n}{2}+k_{0}} \int_{2 \pi t \lambda}^{\infty} J_{\frac{n}{2}+k_{0}}\left(\frac{|m-x|}{t} s\right) J_{\frac{n}{2}+k_{0}+1}(s) d s=0 \quad \text { for } 0 \leq t \leq a .
$$

7.

Therefore by Lebesgue's convergence theorem we conclude the proof of Lemma

In the proof of Lemma 7 we used some properties of the Bessel function.

Property. Suppose $\beta>0$. If $A \geq \beta$ and $\omega>0$, then

$$
\left|\int_{\omega}^{\infty} J_{\alpha-1}(A s) J_{\alpha}(s) d s+\frac{\operatorname{sign}(A-1)}{\pi \sqrt{A}} \int_{|A-1| \omega}^{\infty} \frac{\sin s}{s} d s\right| \leq \frac{C}{\sqrt{A} \omega}\left(1+\frac{1}{\omega}\right),
$$

where $C$ is a constant number depending upon $\beta$ and $\alpha$ only.

In particular

$$
\lim _{\omega \rightarrow \infty} \int_{\omega}^{\infty} J_{\alpha-1}(A s) J_{\alpha}(s) d s=0
$$

and

$$
\left|\int_{\omega}^{\infty} J_{\alpha-1}(A s) J_{\alpha}(s) d s\right| \leq C\left(\frac{1}{\sqrt{A}}+A^{\alpha-1}\right) .
$$

(See Hardy-Landau [3] and Kuratsubo [4].) 
Proof of Theorem 1. Since if $2 \leq n \leq 4$, then $\psi_{1}(\alpha)<\frac{n+1}{4}+\frac{\alpha}{2}$, by Lemma 3 and $\Lambda_{l}\left(t: \lambda^{2}\right)=O\left(\frac{1}{\lambda^{\frac{n+1}{2}+\alpha}}\right)$. Therefore $P_{l}\left(\lambda^{2}: x\right) \Lambda_{l}\left(a: \lambda^{2}\right)$ and

$$
P_{l}\left(\lambda^{2}: x\right) \int_{0}^{a} t^{\frac{n}{2}}(\pi t)^{l} \Lambda_{l}\left(t: \lambda^{2}\right) d \varphi(t)=o(1)
$$

for $0 \leq l \leq k_{0}$. This completes the proof of Theorem 1 .

Proof of Theorem 2. We have the following by Lemma 4:

$$
\psi_{2}(\alpha)<\frac{n+1}{4}+\frac{\alpha}{2} \text { for } \quad \frac{n}{2}-2 \leq \alpha \leq k_{0} .
$$

Therefore we have

$$
\begin{aligned}
P_{\alpha}\left(\lambda^{2}: x\right) \Lambda_{\alpha}\left(a: \lambda^{2}\right) & = \begin{cases}o\left(\lambda^{n-2}\right) O\left(\frac{1}{\lambda^{\frac{n+1}{2}}}\right) & \text { for } \alpha=0, \\
o\left(\lambda^{n-2}\right) O\left(\frac{1}{\lambda^{\frac{n+1}{2}+\alpha}}\right) & \text { for } 0<\alpha<\frac{n}{2}-2, \\
O\left(\lambda^{2 \psi_{2}(\alpha)}\right) O\left(\frac{1}{\lambda^{\frac{n+1}{2}+\alpha}}\right) & \text { for } \frac{n}{2}-2 \leq \alpha \leq k_{0}\end{cases} \\
& =o(1) \quad \text { for } 0 \leq \alpha \leq k_{0}
\end{aligned}
$$

and

$$
P_{\alpha}\left(\lambda^{2}: x\right) \int_{0}^{a} t^{\frac{n}{2}}(\pi t)^{l} \Lambda_{l}\left(t: \lambda^{2}\right) d \varphi(t)=o(1) \quad \text { for } 0 \leq \alpha \leq k_{0} .
$$

This completes the proof of Theorem 2 .

Proof of Theorem 3. If $n \geq 6$, by Lemma 5, we have the following for almost all $x: \psi_{3}(\alpha)<\frac{n+1}{4}+\frac{\alpha}{2}$, for $\alpha \geq 0$, and $\Lambda_{\alpha}\left(t: \lambda^{2}\right)=O\left(\frac{1}{\lambda^{\frac{n+1}{2}+\alpha}}\right)$. Therefore $P_{l}\left(\lambda^{2}: x\right) \Lambda_{l}\left(\lambda^{2}\right)$ and $P_{\alpha}\left(\lambda^{2}: x\right) \int_{0}^{a} t^{\frac{n}{2}}(\pi t)^{l} \Lambda_{l}\left(t: \lambda^{2}\right) d \varphi(t)=o(1)$ for $0 \leq l \leq k_{0}$. This completes the proof of Theorem 3 .

\section{ACKNOWLEDGEMENT}

I would like to thank the referee for sending me the preprint of L. Brandolini and L. Colzani [1] and for helpful suggestions.

\section{REFERENCES}

1. L. Brandolini and L. Colzani, A convergence theorem for multiple Fourier series (preprint).

2. F. Fricker, Einführung in die Gitterpunktlehre, Birkhäuser Verlag, 1982. MR 84b:10001

3. G. H. Hardy and E. Landau, The lattice points of a circle, Proc. Roy. Soc. London Ser. A 105 (1924), 244-258.

4. S. Kuratsubo, On pointwise convergence of Fourier series of the indicator function of $n$ dimensional ball, Sci. Report Hirosaki 43 (1996), 199-208. MR 98e:42010

5. E. Landau, Zur Analytischen Zahlentheorie der definiten quadratischen Formen (Über die Gitterpunkte in einem mehrdimensional Ellipsoid.), Sitzungsber. Kgl. Preuss. Akad. Wiss. 31 (1915), 11-29.

6. B. Novák, Über Gitterpunkte in mehrdimensionalen Ellipsoiden, Czech. Math. J. 22 (1972), 495-507. MR 46:7170

7. M. A. Pinsky, Pointwise Fourier Inversion and Related Eigenfunction Expansions, Comm. Pure and Appl. Math. 47 (1994), 653-681. MR 95e:35145

8. M. A. Pinsky, N. K. Stanton and P. E. Trapa, Fourier Series of Radial Functions in Several Variables, J. Funct. Anal. 116 (1993), 111-132. MR 94j:42019

9. E. M. Stein and G. Weiss, Introduction to Fourier Analysis on Euclidean Spaces, Princeton Univ. Press, 1971. MR 46:4102

Department of Mathematics, Hirosaki University, Hirosaki 036, Japan

E-mail address: kuratubo@cc.hirosaki-u.ac.jp 